\title{
Irritable Bowel Syndrome and Co-morbid Gas- trointestinal and Extra-gastrointestinal Functional Syndromes
}

\author{
Ami D. Sperber, $M D^{1,2 *}$ and Roy Dekel, MD ${ }^{1,3}$ \\ ${ }^{1}$ Department of Gastroenterology, Tel-Aviv Medical Center, Tel-Aviv, Israel, ${ }^{2}$ Faculty of Health Sciences, Ben-Gurion University of the Negev, \\ Beer-Sheva, Israel, ${ }^{3}$ Sackler Faculty of Medicine, Tel-Aviv University, Tel-Aviv, Israel
}

The irritable bowel syndrome (IBS) is the best known of the functional gastrointestinal tract disorders. Many IBS patients have at least one co-morbid somatic complaint and many meet diagnostic criteria for other functional disorders. Patients with IBS and another functional disorder, in comparison with patients with IBS only, have more severe IBS symptoms, a higher rate of psychopathology, greater impairment of quality of life, and more illness-related work absenteeism. Estimates of the prevalence of IBS in patients with fibromyalgia range from $30-35 \%$ to as high as $70 \%$. Studies of IBS among patients with chronic fatigue syndrome have reported a prevalence ranging from 35-92\%. The prevalence of IBS among patients with chronic fatigue syndrome is reported to be $14 \%$. IBS patients with other co-morbid functional disorders appear to manifest a greater degree of somatization. It has been suggested that the presence of multiple co-morbid disorders may be a marker for psychological influences on etiology. This raises the question of whether the functional syndromes represent the same pathophysiological process, i.e., are the same entity that has been separated into different clinical entities because of medical sub-specialization, or are indeed separate disorders. While the answer to this question awaits further research, it would appear that most functional patients who meet formal diagnostic criteria for more than one functional disorder manifest one disorder clinically more that the others and seek consultation differentially for that set of symptoms.

(J Neurogastroenterol Motil 2010;16:113-119)

Key Words

Irritable bowel syndrome, Co-morbidity, Psychopathology, Fibromyalgia

\section{Introduction}

The irritable bowel syndrome (IBS) is the best known of the functional gastrointestinal tract disorders (FGID), all characterized by chronic or recurrent gastrointestinal tract symptoms not explained by structural abnormalities, infection, or metabolic changes on routine testing. ${ }^{1}$ Patients with IBS suffer from chronic abdominal pain, usually in the lower abdomen, with a disturbed bowel pattern, which can be constipation, diarrhea or alternating constipation and diarrhea. Although the range of prevalence rates for IBS in studies conducted throughout the world is broad (from $2-25 \%$ ), in the Western world it is generally agreed that $10-20 \%$ of adults meet diagnostic criteria for IBS. ${ }^{2,3}$ Approximately

Received: February 27th, 2010 Accepted: March 29th, 2010

(c) This is an Open Access article distributed under the terms of the Creative Commons Attribution Non-Commercial License (http://creativecommons. org/licenses/by-nc/3.0) which permits unrestricted non-commercial use, distribution, and reproduction in any medium, provided the original work is properly cited.

*Correspondence: Ami D. Sperber, MD, MSPH

Faculty of Health Sciences, Ben-Gurion University of the Negev, Beer-Sheva, Israel 84105

Tel: +972-8-651-8054, Fax: +972-8-647-7256, E-mail: amy@bgu.ac.il

Financial support: None.

Conflicts of interest: None. 
$60-70 \%$ of IBS patients are women, making it a serious women's health concern.

Systematic reviews of the literature on the co-morbidity of IBS and somatic and psychological disorders have been published over the last decade or so. ${ }^{4,5}$ Whitehead et al. ${ }^{4}$ found that $50 \%$ of IBS patients have at least one co-morbid somatic complaint and many IBS patients meet diagnostic criteria for other functional disorders. There is substantial overlap between IBS and other FGID, such as functional dyspepsia. Significantly, patients with IBS and another functional disorder, in comparison with patients with IBS only, have more severe IBS symptoms, a higher rate of psychopathology such as depression, anxiety and somatization, greater impairment of quality of life, and more illness-related work absenteeism. ${ }^{6,7}$

In another systematic review Riedl et al. ${ }^{5}$ found that IBS patients have a 2-fold increase in co-morbidities, both gastro-

Table 1. Co-morbid Functional Syndromes and Common Unexplained Symptoms

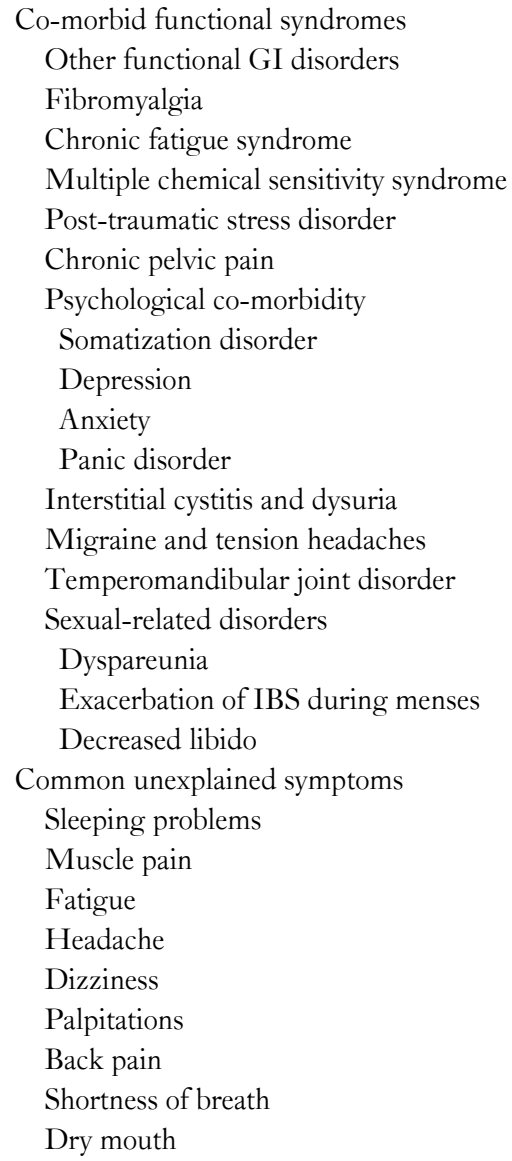

GI, gastrointestinal; IBS, irritable bowel syndrome. intestinal and extraintestinal, and that patients with co-morbidity have more impaired quality of life.

Table 1 presents a list of functional disorders in various body systems and unexplained symptoms that are common in patients with functional disorders and are often considered to be manifestations of multiple system complaints or somatization. ${ }^{8-10}$ The various functional disorders have important characteristics in common, including epidemiology (female predominance), pathophysiology (inflammation, hypersensitivity, impaired central processing of afferent sensory information, role of serotonin, psychological distress and somatization, and the role of stress and life events), diagnosis (symptom-based), the central role of the patient-physician relationship in therapy, and common therapeutic modalities.

The next sections present data on associations between IBS and (1) other gastrointestinal disorders, (2) extraintestinal somatic disorders, and (3) psychological/psychiatric disorders, followed by a brief discussion of possible common pathophysiological mechanisms that underpin these associations.

\section{IBS and Other Functional Gastrointestinal Disorders}

Given the high prevalence of both IBS and functional disorders of the upper gastrointestinal tract, it is not surprising that many patients suffer from more than 1 disorder. Probably the most common association is the one between IBS and functional dyspepsia (FD). A high prevalence of overlap between these 2 conditions has been consistently reported in numerous studies. In a recent meta-analysis, Ford et al. ${ }^{11}$ reported the degree of overlap to be between $15-42 \%$. Moreover people with dyspepsia had an 8 -fold increase in the prevalence of IBS. Another study that used the Rome III criteria found, in 3,014 patients attending a GI clinic, that $24.8 \%$ of FD patients and $31.5 \%$ of IBS patients fulfilled the diagnostic criteria of the other disorder. ${ }^{12}$ In a clinic-based study, Talley et al. ${ }^{13}$ found a significant overlap among IBS, gastroesophageal reflux disease (GERD), and FD. The overlap between IBS and FD was over $80 \%$. In sub-group analyses patients with constipation-predominant IBS had higher rates of overlap than patients with diarrhea-predominant IBS.

Furthermore long-term follow ups of patients with IBS and FD demonstrate that over time patients with IBS develop FD symptoms and vice versa. In a 1-year follow-up, $22 \%$ of patients with IBS changed their symptom profile from FD to IBS. ${ }^{14}$ These 2 entities also share a similar profile of patients (female 
predominance, high levels of psychological co-morbidities) and pathophysiologic mechanisms, mainly visceral hypersensitivity. Therefore some argue that the distinction between these 2 entities might be artificial and that they actually represent 1 syndrome with different clinical presentations. ${ }^{15}$

Another common association of IBS is with GERD. Nastaskin et al. ${ }^{16}$ conducted a systematic review of the literature to assess associations between IBS and GERD. They reported that the overlap between them is significantly greater than their individual prevalence rates and the rates for each disorder would be significantly lower if individuals with overlap were removed from analyses. Two other population based studies in the United States and the United Kingdom reported 25\% and 19\% overlap between IBS and GERD, respectively. ${ }^{17,18}$ A population-based study found a considerable overlap rate between IBS, FD, and GERD. Twenty one percent of IBS patients also had GERD symptoms. Patients with symptom overlap demonstrated high levels of anxiety compared with patients with only 1 FGID. ${ }^{19}$ Another study found 36 percent prevalence of IBS in GERD patients. In addition, the concomitant presence of IBS or psychological distress in GERD patients predicted higher symptom ratings and lower quality of life before and after PPI treatment, making this subset of patients a therapeutic challenge. ${ }^{20}$ As in functional dyspepsia, visceral hyperalgesia could be a unifying underlying mechanism that could explain the high prevalence of overlap. $^{21}$

\section{IBS and Other Extraintestinal Disorders -}

Fibromyalgia syndrome (FMS), chronic fatigue syndrome (CFS), chronic pelvic pain (CPP), sexual dysfunction, and sleep disturbances are non-gastrointestinal functional disorders whose associations with IBS have drawn the greatest amount of research interest. The studies are consistent in their finding that the overlap among these disorders is greater than expected based on their separate prevalence rates. In addition, patients with more than 1 disorder have greater disease severity, higher rates of psychopathology, and more severely impaired health-related quality of life than those with only 1 disorder. ${ }^{4}$

Inasmuch as the functional disorders overlap significantly and have similarities in terms of patient characteristics, impact of illness on quality of life, and psychological co-morbidity and treatment, it has been suggested that they may represent a group of illnesses sharing a common pathogenesis. ${ }^{22-24}$ In fact, they are so similar in many respects that some authorities have suggested that their classification into separate entities is artificial; ${ }^{25}$ a result of the division of modern medicine into medical sub-specialties. This issue will be discussed below. The following sections present evidence of associations between IBS and several extraintestinal functional disorders.

\section{Fibromyalgia syndrome}

FMS is a soft tissue disorder characterized by diffuse musculoskeletal pain and specific tender points on examination, ${ }^{26}$ without evidence of synovitis or myositis.

Estimates of the prevalence of IBS in patients with FMS range from $30-35 \%^{27}$ to as high as $70 \%{ }^{22}$ Using consensus criteria, our group diagnosed FMS in $31.6 \%$ of IBS patients compared to $4.2 \%$ of controls. Correspondingly, IBS was diagnosed in $32 \%$ of women with known FMS. $^{28}$ In all clinical and health-related variables assessed, including global feeling of wellbeing, sleep disturbances, relation of stress to symptoms, physician visits, concerns about illness, and psychological distress, patients with both IBS and FMS had more severe disorders than those with IBS only. The same pattern held true for the Sense of Coherence Index (a measure of coping ability) ${ }^{29}$ and the Functional Bowel Disorder Severity Index. ${ }^{30}$

FMS is considered a disorder of somatic hypersensitivity, while IBS is considered a disorder of visceral hypersensitivity. Although IBS patients have been reported to have lower, rather than higher thresholds for somatic stimulation, ${ }^{11,12}$ Chang et al. ${ }^{31}$ reported that female IBS patients with co-morbid FMS have increased sensitivity to somatic stimuli, while those with IBS without FMS show a blunted response.

\section{Chronic fatigue syndrome}

The prevalence of CFS in the general population is reported to be $0.4 \% .^{32} \mathrm{Up}$ to $70 \%$ of FMS patients have $\mathrm{CFS}^{33}$ and $35-70 \%$ of patients with CFS have FMS. ${ }^{33,34}$ Studies of IBS among CFS patients have reported a prevalence ranging from $35-92 \%{ }^{34-38}$ with a median of $51 \%$. $^{4}$ The prevalence of IBS among patients with CFS is reported to be $14 \% .^{35}$

Aaron et al. ${ }^{38}$ reported the results of a co-twin control study of 127 chronic fatigue patients. Co-morbid conditions such as FMS, IBS, tempero-mandibular joint pain (TMJ), interstitial cystitis, chronic pelvic pain (CPP), tension headache, multiple chemical sensitivities, and other functional disorders were significantly more prevalent in the twin with CFS than in the non-fatigued one. 


\section{Chronic pelvic pain}

The prevalence of CPP in IBS was reported to be $14 \% .{ }^{39}$ The rates of IBS in women with CPP are even higher, ranging from $29-79 \%{ }^{39,40}$ Williams et al. ${ }^{41}$ studied 987 new patients referred to a CPP clinic and reported that $35 \%$ met the Rome I criteria for IBS, although IBS was not diagnosed previously in $40 \%$ of these patients. Longstreth et al. ${ }^{42}$ assessed 86 women undergoing gynecological laparoscopy for CPP. IBS was diagnosed in $47.7 \%$ of these women.

The results of studies have shown that a greater awareness of IBS on the part of gynecologists could reduce the rate of unnecessary diagnostic tests and procedures in women complaining of pelvic/lower abdominal pain. Because of the similarity in pain of bowel and gynecological origin, many IBS patients undergo gynecological work-ups, including pelvic laparoscopy for pelvic pain, without any pathological findings.

\section{Sexual dysfunction}

Several studies have reported an increased prevalence of sexual dysfunction among IBS patients, including reduced sexual drive, ${ }^{43}$ increased dyspareunia, ${ }^{39,44}$ and more severe IBS symptoms following intercourse. IBS patients report a high rate of intercourse avoidance. ${ }^{45}$ IBS symptoms worsen during menses. ${ }^{46}$ The most common sexual dysfunction in IBS patients may be decreased libido. Fass et al. ${ }^{43}$ reported decreased sexual drive in $36.2 \%$ of male patients and $28.4 \%$ of female patients. The prevalence of any reported sexual dysfunction among male and female patients was $40.6 \%$, which was significantly higher than among controls (OR, 4.33; 95\% CI, 2.69-7.25; p < 0.001). Among women with IBS with a sexual problem over the previous six months, 36\% reported a decreased sexual drive, 32\% reported dyspareunia, $32 \%$ reported that bowel symptoms directly prevented them from engaging in intercourse, and $15 \%$ reported that sexual dysfunction worsened directly in relation to the severity of their bowel problems.

Prior et al. ${ }^{47}$ evaluated 798 patients referred by general practitioners to a gynecological clinic over a 6 year period. Among the 63 women who were referred for dyspareunia, $52.4 \%$ were found to meet diagnostic criteria for IBS (95\% CI, 40.8-59.2; p < $0.001)$

\section{IBS and psychological/psychiatric co-morbidity}

There is a recognized overlap between IBS and psychiatric disorders that involves a large percentage of IBS patients. ${ }^{48}$ The percentage is particularly high among patients seen in tertiary referral centers and lower in IBS patients seen and treated in the community setting. ${ }^{49}$ However, as discussed by North et al. ${ }^{10}$ most IBS patients do not have psychiatric illness per se but do manifest psychoform symptoms and somatoform complaints.

Lydiard assessed FGID in 73 panic disorder patients and compared these rates to the United States Household Survey. ${ }^{7}$ He reported higher rates of IBS and other FGIDs in these patients than in the general public. ${ }^{50}$ The mean number of FGIDs was 3.9 in the panic disorder group, so the co-morbidity encompasses many FGIDs and not only IBS.

Gros et al. ${ }^{51}$ studied 357 patients with anxiety and depressive disorders and found elevated levels of co-morbid IBS symptoms in patients with general anxiety disorder (GAD), panic disorder, and major depression. Patients who met the Rome II criteria for IBS had increased general anxiety compared to those without.

Recently the results of community-based studies have been published. Lee et al. ${ }^{52}$ studied the co-morbidity of IBS and GAD in a community-based sample of 2,005 adolescents and adults from Hong Kong. They showed an overall prevalence of 5.4\% for IBS using the Rome III diagnostic criteria and $4.0 \%$ for GAD..$^{52}$ The OR for IBS was 5.84 among respondents with GAD compared to those without GAD and the OR for GAD was 6.32 for respondents with IBS compared to those without it (both, $\mathrm{p}<0.001$ ), showing considerable co-morbidity between these disorders.

An aspect of psychiatric co-morbidity that appears to be of particular significance in the study of IBS and associated functional disorders is somatization disorder (SD). North et al. ${ }^{8}$ reported a very high rate of non-gastrointestinal functional disorders in IBS patients, but significantly more so among those with SD. They reported that many aspects of co-morbidity and health care behaviors were more associated with the diagnosis of $\mathrm{SD}$ than with the diagnosis of IBS. In fact SD was much more important that depression or anxiety, which are often considered as psychiatric associates of IBS. This may not be surprising given that $\mathrm{SD}$ is defined, in part, as the manifestation of multiple symptoms across different organ systems.

Whitehead et al. have suggested that IBS patients with other co-morbid functional disorders manifest a greater degree of somatization, which may serve as a link between these disorders and increased health care utilization, disability, and impaired quality of life. ${ }^{54}$ They propose that the presence of multiple co-morbid disorders is a marker for psychological influences on etiology. ${ }^{4}$ This link has potential therapeutic ramifications. 


\section{One syndrome, or many?}

In the mid 19th century Beard, ${ }^{55}$ an American neurologist, published a paper on "neurasthenia", a term he used to describe individuals with unexplained chronic fatigue and lassitude (nervous exhaustion). He noted that these patients had additional symptoms including sleep difficulties, back pain, nervousness, anxiety, depression, headache, difficulty in concentrating, reduced sexual impulse, abdominal pain, bloating and excess gas, diarrhea, and lack of appetite. Although he described patients of both sexes, he reported a female predominance. The patients he described might be diagnosed today with CFS, or depending on the specialist that saw them, any of the other system-specific functional disorders.

Others have suggested that the disorders may be related through their association with depression and have suggested classifying them as a single entity called the "affective spectrum disorder." 24,56 However, Henningsen et al. ${ }^{57}$ performed a meta-analysis on the association between medically unexplained physical symptoms, anxiety, and depression and concluded that while IBS, FMS, and CFS are associated with anxiety and depression, they are not dependent on these psychological dimensions and cannot be classified as "common mental disorders." Still, as discussed above, SD is a potentially important link between the various functional disorders.

So are the functional disorders 1 syndrome or many? The jury is still out on this intriguing issue. While the name of the syndrome may be determined by the specialist who sees the patient, as has been suggested and discussed above, a more relevant question may be why did the patient see that particular specialist? In our experience, patients who meet the diagnostic criteria for several functional disorders often manifest 1 disorder clinically much more that the others and seek consultation differentially for that set of symptoms. Thus, a patient could be diagnosed formally with CPP, IBS, FMS, and TMJ, but judging by their consultation pattern might be considered a patient of 1 syndrome and a non-patient in the others. For example, a patient diagnosed with both IBS and FMS might be an IBS-patient and an FMS non-patient, or an FMS-patient and an IBS non-patient. In the first instance they would likely consult a gastroenterologist and in the second a rheumatologist. Our clinical impression is that many patients with more that 1 formal diagnosis do not consult multiple specialists, but concentrate on the physician who specializes in the symptoms that they suffer from in particular.
In this respect it is interesting that Wessely, who was the first author of the paper that discussed the potentially artifactual separation of functional syndromes into separate entities, ${ }^{25}$ states in a 2007 paper that: "In the functional somatic disorders, a diagnosis that represents the patient's view of his/her illness stands to be both instrumentally and pragmatically apt." ${ }^{58}$ This would seem to be particularly appropriate for disorders that are diagnosed on the basis of patients' subjective symptom reports rather than on the basis of objective tests.

In summary, many IBS patients have at least 1 co-morbid somatic complaint and many of them meet diagnostic criteria for other gastrointestinal and extraintestinal functional disorders. Patients with IBS and 1 or more other functional disorders, in comparison with patients with IBS only, have more severe IBS symptoms, a higher rate of psychopathology such as depression, anxiety and somatization, and a greater impairment of quality of life.

\section{References}

1. Drossman DA, Camilleri M, Mayer EA, Whitehead WE. AGA technical review on irritable bowel syndrome. Gastroenterology 2002;123:2108-2131.

2. Cremonini F, Talley NJ. Irritable bowel syndrome: epidemiology, natural history, health care seeking and emerging risk factors. Gastroenterol Clin N Amer 2005;34:189-204.

3. Hungin AP, Whorwell PJ, Tack J, Mearin F. The prevalence, patterns and impact of irritable bowel syndrome: an international survey of 40,000 subjects. Aliment Pharmacol Ther 2003;17:643-650.

4. Whitehead WE, Palsson O, Jones KR. Systematic review of the comorbidity of irritable bowel syndrome with other disorders: what are the causes and implications? Gastroenterology 2002;122:1140-1156.

5. Riedl A, Schmidtmann M, Stengel A, et al. Somatic comorbidities of irritable bowel syndrome: a systematic analysis. J Psychosom Res 2008;64:573-582.

6. Markowitz M, Harris W, Ricci JF, et al. Comorbid conditions in patients with irritable bowel syndrome: data from a national IBS awareness registry. Gastroenterology 2001;120(suppl 1):105.

7. Drossman DA, Li Z, Andruzzi E, et al. U.S. householder survey of functional GI disorders: prevalence, sociodemography and health impact. Dig Dis Sci 1993;38:1569-1580.

8. North CS, Downs D, Clouse RE, et al. The presentation of irritable bowel syndrome in the context of somatization disorder. Clin Gastroenterol Hepatol 2004;2:787-795.

9. Miller AR, North CS, Clouse RE, Wetzel RD, Spitznagel EL, Alpers DH. The association of irritable bowel syndrome and somatization disorder. Ann Clin Psychiatry 2001;13:25-30.

10. North CS, Hong BA, Alpers DH. Relationship of functional gastrointestinal disorders and psychiatric disorders: implications for treatment. World J Gastroenterol 2007;13:2020-2027.

11. Ford AC, Marwaha A, Lim A, Moayyedi P. Systematic review and 
meta-analysis of the prevalence of irritable bowel syndrome in individuals with dyspepsia. Clin Gastroenterol Hepatol 2009:Epub.

12. Wang A, Liao X, Xiong $\mathrm{L}$, et al. The clinical overlap between functional dyspepsia and irritable bowel syndrome based on Rome III criteria. BMC Gastroenterol 2008;8:43.

13. Talley NJ, Dennis EH, Schettler-Duncan VA, Lacy BE, Olden KW, Crowell MD. Overlapping upper and lower gastrointestinal symptoms in irritable bowel syndrome patients with constipation or diarrhea. Am J Gastroenterol 2003;98:2454-2459.

14. Agreus L, Svardsudd K, Nyren O, Tibblin G. Irritable bowel syndrome and dyspepsia in the general population: overlap and lack of stability over time. Gastroenterology 1995;109:671-680.

15. Gwee KA, Chua AS. Functional dyspepsia and irritable bowel syndrome, are they different entities and does it matter? World J Gastroenterol 2006;12:2708-2712.

16. Nastaskin I, Mehdikhani E, Conklin J, Park S, Pimentel M. Studying the overlap between IBS and GERD: a systematic review of the literature. Dig Dis Sci 2006;51:2113-2120.

17. Locke GR 3rd, Zinsmeister AR, Talley NJ, Fett SL, Melton LJ 3rd. Familial association in adults with functional gastrointestinal disorders. Mayo Clin Proc 2000;75:907-912.

18. Kennedy TM, Jones RH, Hungin APS, O'Flanagan H, Kelly P. Irritable bowel syndrome, gastro-oesophageal reflux, and bronchial hyper-responsiveness in the general population. Gut 1998;43:770774.

19. Lee SY, Lee KJ, Kim SJ, Cho SW. Prevalence and risk factors for overlaps between gastroesophageal reflux disease, dyspepsia, and irritable bowel syndrome: a population-based study. Digestion 2009;79: 196-201.

20. Nojkov B, Rubenstein JH, Adlis SA, et al. The influence of co-morbid IBS and psychological distress on outcomes and quality of life following PPI therapy in patients with gastro-oesophageal reflux disease. Aliment Pharmacol Ther 2008;27:473-482.

21. Dickman R, Feroze H, Fass R. Gastroesophageal reflux disease and irritable bowel syndrome: a common overlap syndrome. Curr Gastroenterol Rep 2006;8:261-265.

22. Veale D, Kavanagh G, Fielding JF, Fitzgerald O. Primary fibromyalgia and the irritable bowel syndrome: different expressions of a common pathogenetic process. Br J Rheumatol 1991;30:220-222.

23. Gruber AJ, Hudson JI, Pope HG Jr. The management of treatment-resistant depression in disorders on the interface of psychiatry and medicine. Psychiatr Clin North Am 1996;19:351-369.

24. Hudson JI, Goldenberg DL, Pope HGJ, Keck PEJ, Schlesinger L. Comorbidity of fibromyalgia with medical and psychiatric disorders. Am J Med 1992;92:363-367.

25. Wessely S, Nimnuan C, Sharpe M. Functional somatic syndromes: one or many? Lancet 1999;354:936-939.

26. Wolfe F, Smythe HA, Yunus MB, et al. The American College of Rheumatology 1990 criteria for the classification of fibromyalgia. Report of the multicenter criteria committee. Arthritis Rheum 1990; 33:160-172.

27. Pace F, Manzionna G, Bollani S, Sarzi-Puttini P, Bianchi Porro G. Visceral sensitivity in patients with fibromyalgia and in normal patients [abstract]. Gastroenterology 1997;112:A802.

28. Sperber AD, Atzmon Y, Neumann L, et al. Fibromyalgia in the irritable bowel syndrome: studies of prevalence and clinical implications.
Am J Gastroenterol 1999;94:3541-3546.

29. Sperber AD, Carmel S, Atzmon Y, et al. The sense of coherence index and the irritable bowel syndrome. A cross-sectional comparison among irritable bowel syndrome patients with and without coexisting fibromyalgia, irritable bowel syndrome non-patients, and controls. Scand J Gastroenterol 1999;34:259-263.

30. Sperber AD, Carmel S, Atzmon Y, et al. Use of the Functional Bowel Disorder Severity Index (FBDSI) in a study of patients with the irritable bowel syndrome and fibromyalgia. Am J Gastroenterol 2000;95:995-998.

31. Chang L, Berman S, Mayer EA, et al. Brain responses to visceral and somatic stimuli in patients with irritable bowel syndrome with and without fibromyalgia. Am J Gastroenterol 2003;98:1354-1361.

32. Jason LA, Richman JA, Rademaker AW, et al. A community-based study on chronic fatigue syndrome. Arch Intern Med 1999;159: 2129-2137.

33. Buchwald D, Garrity D. Comparison of patients with chronic fatigue syndrome, fibromyalgia, and multiple chemical sensitivities. Arch Intern Med 1994;154:2049-2053.

34. Goldenberg DL, Simms RW, Geiger A, Komaroff AL. High frequency of fibromyalgia in patients with chronic fatigue seen in a primary care practice. Arthritis Rheum 1990;33:381-387.

35. Jones KR, Palsson OS, Levy RL, et al. Comorbid disorders and symptoms in irritable bowel syndrome (IBS) compared to other gastroenterology patients [abstract]. Gastroenterology 2001;120(suppl 1):A66.

36. Gomborone JE, Gorard DA, Dewsnap PA, Libby GW, Farthing MJ. Prevalence of irritable bowel syndrome in chronic fatigue. J R Coll Physicians Lond 1996;30:512-513.

37. Aaron LA, Burke MM, Buchwald D. Overlapping conditions among patients with chronic fatigue syndrome, fibromyalgia, and temporomandibular disorder. Arch Intern Med 2000;160:221-227.

38. Aaron LA, Herrell R, Ashton S, et al. Comorbid clinical conditions in chronic fatigue: a co-twin control study. J Gen Intern Med 2001;16:24-31.

39. Walker EA, Katon WJ, Jemelka R, Alfrey H, Bowers M, Stenchever MA. The prevalence of chronic pelvic pain and irritable bowel syndrome in two university clinics. J Psychosom Obstet Gynaecol 1991;12(suppl):65-75.

40. Longstreth GF. Irritable bowel syndrome and chronic pelvic pain. Obstet Gynecol Survey 1994;49:505-507.

41. Williams RE, Hartmann KE, Sandler RS, Miller WC, Savitz LA, Steege JF. Recognition and treatment of irritable bowel syndrome among women with chronic pelvic pain. Am J Obstet Gynecol 2005;192:761-767.

42. Longstreth G, Preskill DB, Youkeles L. Irritable bowel syndrome in women having diagnostic laparoscopy or hysterectomy. Relation to gynecologic features and outcome. Dig Dis Sci 1990;35:1285-1290.

43. Fass R, Fullerton S, Naliboff B, Hirsh T, Mayer EA. Sexual dysfunction in patients with irritable bowel syndrome and non-ulcer dyspepsia. Digestion 1998;59:79-85.

44. Whorwell PJ, McCallum M, Creed FH, Roberts CT. Non-colonic features of irritable bowel syndrome. Gut 1986;27:37-40.

45. Corney RH, Stanton R. Physical symptom severity, psychological and social dysfunction in a series of outpatients with irritable bowel syndrome. J Psychosom Res 1990;34:483-491. 
46. Whitehead WE, Cheskin LJ, Heller BR, et al. Evidence for exacerbation of irritable bowel syndrome during menses. Gastroenterology 1990;98:1485-1489.

47. Prior A, Wilson K, Whorwell PJ, Faragher EB. Irritable bowel syndrome in the gynecological clinic. Survey of 798 new referrals. Dig Dis Sci 1989;34:1820-1824.

48. Lydiard RB, Fossey MD, Marsh W, Ballenger JC. Prevalence of psychiatric disorders in patients with irritable bowel syndrome. Psychosomatics 1993;34:229-234.

49. Walker EA, Katon WJ, Jemelka R, Roy-Byrne PP. Comorbidity of gastrointestinal complaints, depression and anxiety in the Epidemiological Catchment Area (ECA) study. Am J Med 1992;92(suppl 1A):26S-30S.

50. Lydiard RB. Increased prevalence of functional gastrointestinal disorders in panic disorder: clinical and theoretical implications. CNS Spectr 2005;10:899-908.

51. Gros DF, Antony MM, McCabe RE, Swinson RP. Frequency and severity of the symptoms of irritable bowel syndrome across the anxiety disorders and depression. J Anxiety Disord 2009;23:290-296.

52. Lee S, Wu J, Ma YL, Tsang A, Guo WJ, Sung J. Irritable bowel syndrome is strongly associated with generalized anxiety disorder: a community study. Aliment Pharmacol Ther 2009;30:643-651.

53. Choung RS, Locke GR 3rd, Zinsmeister AR, Schleck CD, Talley NJ. Psychosocial distress and somatic symptoms in community subjects with irritable bowel syndrome: a psychological component is the rule. Am J Gastroenterol 2009;104:1772-1779.

54. Palsson OS, Jones KR, Turner M, Drossman DA, Whitehead WE. Impact of somatization and comorbid medical conditions on health care utilization, disability, and quality of life in irritable bowel syndrome (IBS) [abstract]. Gastroenterology 2002;122:A501-A502.

55. Beard GM. Neurasthenia, or nervous exhaustion. Bost Med Surg J 1869;80:217-221.

56. Hudson JI, Pope HG Jr. Affective spectrum disorder: does antidepressant response identify a family of disorders with a common pathophysiology? Am J Psychiatry 1990;147:552-564.

57. Henningsen P, Zimmermann T, Sattel H. Medically unexplained physical symptoms, anxiety, and depression: a meta-analytic review. Psychosom Med 2003;65:528-533.

58. Kanaan RA, Lepine JP, Wessely SC. The association or otherwise of the functional somatic syndromes. Psychosom Med 2007;69:855859 